\title{
COMPETENCIAS INTERCULTURALES EN SERVICIOS DE ORIENTACIÓN PARA EL DESARROLLO DE LA CARRERA
}

\author{
INTERCULTURAL COMPETENCIES AT CAREER \\ DEVELOPMENT COUNSELLING SERVICES
}

\author{
José Luis Castellano Pérez* \\ Universidad Nacional de Educación a Distancia
}

\begin{abstract}
RESUMEN
Se presenta un modelo de análisis de competencias interculturales en el área de conocimiento de la orientación adaptado a los servicios de orientación en universidades a distancia europeas. Tanto servicios como profesionales trabajando en ellos son foco del análisis. En primer lugar, se procede a un acercamiento al concepto de competencia. En segundo lugar, se comenta lo que desde el contexto norteamericano se ha hecho en orientación para el desarrollo de la carrera y se comenta el estado actual de la investigación en orientación intercultural. A continuación se presenta un modelo de competencias interculturales, explicamos su necesidad y comentamos brevemente los resultados de la investigación en torno a este tópico en Europa.
\end{abstract}

Palabras clave: Competencia, competencias interculturales, desarrollo de la carrera, orientación intercultural.

\begin{abstract}
It is presented a model of intercultural competencies analysis within the general knowledge area of counselling focused on counselling services at European distance universities. Services as well as practitioners working in them are themes of the analysis. First of all, it is presented a competence concept. On the other hand, it is commented what from the North American context has build up in career counselling. Third, it is offered an insight of the research situation in intercultural counselling. Finally, it is presented an intercultural model of competencies and it is briefly explained and commented last results of the research on this topic.
\end{abstract}

Key words: Competence, intercultural competences, career development, intercultural ounselling.

* José Luis Castellano es Licenciado y Doctor en Ciencias de la Educación, por la Universidad Nacional de Educación a Distancia. Línea de investigación actual: Competencias en Orientación Intercultural. 


\section{Introducción}

La carrera de los individuos depende en gran medida de lo que les ofrece el entorno. No es lo mismo crecer en un contexto institucional rico en recursos que hacerlo en uno pobre. Existen también grandes diferencias en las oportunidades que ofrecen los entornos institucionales a la adquisición de estrategias de desarrollo personal. Si tenemos en cuenta que los individuos dependen de los servicios, es importante reflexionar sobre la responsabilidad de éstos en el desarrollo de las personas que comparten un mismo lugar geográfico y sociopolítico. Nosotros partimos de la premisa de que todas las personas tienen derecho a la planificación de aquellos caminos vitales que más les interesen de acuerdo con sus características personales y sociales. También se es consciente de que no se parte de la nada y de que hoy por hoy se está asentado en situaciones institucionales que no siempre responden a las demandas de sus usuarios. La nueva situación económica exige a los ciudadanos, y también a las instituciones, adaptarse a los continuos cambios que se dan. Por ejemplo, desde la OCDE (estudio PISA, 2000) se recomienda encarecidamente a los sistemas educativos, que son los que filtran en gran medida las posiciones sociales y caminos vitales, que reduzcan su poder selectivo para convertirse en sistemas que ofrezcan salidas y oportunidades para todos.

Cada contexto institucional tiene su granito de responsabilidad. El artículo se centra en la educación a distancia superior, con un grado de heterogeneidad importante, y dentro de ésta, en los servicios de orientación, una parcela poco explorada. Generalmente se declara que se orienta por igual a todos los usuarios, pensando que con esto se va a ayudar por igual a todos. En el artículo se parte de otra premisa: no se puede orientar igual en el estudio a distancia a una mujer madre de familia de 40 años que necesita incorporarse al mundo laboral, que a un hombre y profesional ya cualificado que quiere enriquecer sus competencias para adaptarse a los cambios y necesidades de su empresa. Los servicios de orientación tendrían que estar provistos de las competencias necesarias para ayudar a todos, pero no por igual, sino según sus necesidades.

El texto ofrece un modelo de competencias interculturales básicas a desarrollar por servicios y profesionales de la orientación para el desarrollo de la carrera en universidades a distancia en Europa. El documento es resultado de la investigación cualitativa que se ha llevado a cabo en cinco universidades europeas a distancia (Fernuniversität en Alemania, Open Universiteit de Holanda, Open University de Gran Bretaña, Universidade Aberta de Portugal y UNED de España).

De la revisión bibliográfica realizada, no existe un modelo de desarrollo de competencias interculturales único, ni, por consiguiente, una base teórica intercultural única. El modelo que se presenta es resultado del consenso hallado entre diferentes autores de diferentes contextos históricos, políticos, geográficos y lingüísticos (Aguado et al., 1998; Arredondo, et al., 1996; Auernheimer, 2002, Dekelskamp, 1991; Palmer, C. \& Laungani, P. 1999; Hammer et al., 1978; Hannigan, 1991; Herbrand, F. 2000; Hoffmann, E.; 2002, Ruben, 1990; Sodowsky, et al., 1994; Sue, et al. 1992, 1998; Sue \& Sue 1999 entre otros).

\section{Aproximación al concepto de competencia}

Acudimos a Baitsch, C. (1985); Frei, Duell \& Baitsch (1984), Frei, et al. (1986), autores que trabajan en la línea de Leontiev y de Vygotsky. Para Leontiev (1973) la actividad prác- 
tica gobernada por la actividad psíquica e impulsada por la motivación hacia una meta genera una conciencia en forma de representaciones. Por otro lado, la conciencia es dependiente de las condiciones materiales e históricas en continuo cambio. Sin embargo, las representaciones son, en primer lugar, consecuencia de la actividad, consecuencia de la indagación orientada y analítica (actividad interna) del sujeto, que se concreta en la acción (actividad externa) (Baitsch, 85: 31 y ss.).

Para Leontiev el trabajo es la actividad humana clave responsable de experiencia histórica. El trabajo tiene dos características fundamentales:

a) mediante el trabajo el ser humano desarrolla y utiliza herramientas,

b) además, supone una actividad colectiva.

Mediante la experiencia activa a través del trabajo es posible adquirir nuevos conocimientos $\mathrm{y}$, sobre todo, nuevos significados, fruto de la transformación de las representaciones o conciencia.

Los significados contienen las experiencias de las generaciones pasadas así como de las presentes en forma de sistemas de conceptos, conocimientos y destrezas socialmente construidos. Los significados se dan, en parte, independientemente de los individuos, puesto que son fenómenos que se van desarrollando históricamente en los grupos; así pues, las personas estamos y nos movemos en sistemas de significados que nos preceden y aprendemos. A su vez los significados son elaborados de manera activa y reconstruidos por las personas, por los individuos imbuidos socialmente. La relación entre significado y sentido personal comprende la relación entre los componentes principales de la estructura interna de la conciencia humana. Por otro lado, la capacidad de modificación de actividad interna hace posible y necesita de la apropiación de significados como construcción de la realidad. Esta modificabilidad de la actividad psíquica es lo que constituye el sistema aprendiente.

La actividad psíquica es un sistema que funciona a partir de una serie de capacidades, destrezas y conocimientos filtrados por valores y actitudes adquiridos a través de la experiencia y provocada por la actividad dirigida por necesidades, motivos y metas. La competencia es la relación sistémica de estos factores, actualizada en una actividad concreta por un individuo de modo diferencial. Necesidades y metas motivan para la acción, lo que conduce a determinadas experiencias. Mediante estas experiencias desarrolla el individuo saberes, capacidades y destrezas. Las experiencias proporcionan también valores y actitudes, que determinan las metas e intereses del individuo. Este proceso se desarrolla durante toda la vida (Frei et al., 1996: 17).

La fuente del desarrollo de competencias se halla en dos tipos de condicionantes: por un lado, en la posibilidad misma de desarrollo de competencias, las oportunidades que el contexto institucional proporcione al individuo para desarrollar las competencias, esto es, las oportunidades socialmente reguladas que el contexto institucional proporcione a los individuos para la reconstrucción de significados sociales (saberes, capacidades, destrezas, valores, normas, tecnologías, etc.). Por otro lado, el desarrollo de competencias depende de la sensibilidad y voluntad de reconstrucción de nuevos significados, lo cual viene determinado por las experiencias previas en este sentido que haya tenido el individuo (Frei et al. 1996: 19). Los procesos de aprendizaje son, o bien individuales, o bien colectivos. Los procesos de aprendizaje individual se desarrollan a través del crecimiento (novedades), diferenciación (análisis) e integración (síntesis); y los sistémicos o colectivos a través de desenlace (auftauen, fruto del desarrollo de fuerzas sociales contrarias), cambio (innovaciones), con- 
solidación (reflexión: la innovación es experimentada como provechosa). Este proceso de cambio competencial exige la participación directa y organizada de los afectados en los procesos de gestión del mismo (Frei et al. 1996: 16-31).

Las dimensiones del desarrollo o cambio de las competencias son:

Extensión: contenido y grado de organización de tareas que tiene el sujeto; esto es, extensión en la organización subjetiva de las clases de actividad (las competencias se refieren a la actividad; el objeto de la actividad es el motivo hacia el que se dirige la actividad; si cambian los motivos se modifica la extensión de la competencia). Intención: sentido individual (según necesidades, dirigidas por motivos y metas) o sentido colectivo (grupal y organizacional). Reflexión: calidad de la elaboración lingüística-conceptual, o grado de generalización conceptual de las características de la tarea (Baitsch 1996: 3-11).

En el marco de cualquier profesión, el desarrollo ontogenético de las competencias ocurre en el transcurso de actividades concretas. Este proceso de actualización del desarrollo de las competencias es denominado cualificación. La probabilidad estadística de que ocurra un proceso de cualificación es el potencial de cualificación. Este está causado por tipos de determinantes: oferta de cualificación (determinante objetivo) y disposición (subjetivo).

Estas dimensiones son analizadas también para grupos y organizaciones. En cuanto a los primeros hay tres tipos de características estructurales:

a) metas comunes;

b) diferenciación de roles, relaciones específicas en torno a tareas y cooperación, a través de la cual se formalizan experiencias comunes;

c) desarrollo de una cultura común en torno a un conjunto de valores y normas comunes, con lo que se genera un significado colectivo específico.

La calidad de los procesos de gestión y dirección colectivos se genera y desarrolla de modo análogo al desarrollo individual. La extensión de la competencia se determina a partir de la definición de la actividad colectiva, el ámbito de intención de la competencia de grupos se relaciona con la génesis de sentido colectivo. Por último, el ámbito de reflexión de la competencia de grupos se refiere a la autotematización como grupo. En estos dos últimos ámbitos, los procesos de comunicación son fundamentales. En el ámbito de la intención se trata del problema de la toma de decisiones, mientras que en el segundo el grupo necesita de una imagen propia construida en base a procesos comunicativos verbales y no verbales tanto entre los miembros del grupo, como con el exterior.

La competencia de las organizaciones es definida como la calidad de las muestras de gestión referidas a la autodefinición y saber organizacionales. El ámbito de la extensión de la competencia se refiere en este caso a la extensión y complejidad de las funciones a desempeñar por los miembros de la organización. La pregunta es, ¿según qué medidas desempeña una organización la formación de tareas específicas y qué medidas toma para garantizar la posibilidad de funciones laborales? Esto se desprende de la política de cualificaciones de la organización: formación y formación permanente, por un lado, medidas de gestión, esto es, procesos de aprendizaje laboralmente inmanentes (sistema de salarios, formación de tareas, etc.). El ámbito de intención competencial se refiere al marco de referencia organizacional para la toma de decisiones en y sobre la organización. Se trata del sentido de la organización como organización. El ámbito de reflexión competencial en las organizaciones 
se refiere a las actividades y artefactos de las organizaciones, que afectan a la producción de la organización. Se trata en primera instancia del autoconcepto de la organización.

Esta línea teórica acerca de las competencias (Leontiev, Frei et al. y Baitsch) me parece interesante para nuestro propósito, porque inserta las competencias en un marco cultural (representaciones), las sitúa a lo largo de toda la vida, siendo resultado de un proceso de aprendizaje o cambio en cualquier momento según las necesidades individuales y/o sociales, y establece un marco de gestión en el que todos los participantes o actores sociales tienen que o pueden formar parte activamente en el cambio, si es que éste se pretende sea duradero (proceso de consolidación) y lo suficientemente flexible como para volver a cambiar. La política de cualificaciones se gestiona a tres niveles competenciales: a nivel organizacional (institucional); los grupos desempeñan la función que es planificada en el nivel anterior. Esta función la internaliza el individuo diferencialmente. Es interesante, porque desde esta perspectiva las competencias multiculturales no son asunto de los individuos en exclusiva. En este caso se desenfocaría las coordenadas del trabajo humano. En la revisión bibliográfica efectuada sobre competencias se carga el acento en la persona, en el profesional, no en los grupos, y mucho menos en las organizaciones, aunque una de las competencias sea ser consciente de la historia de opresión y racismo institucional.

\section{Evolución de la orientación para el desarrollo de la carrera}

Nos vamos a basar en dos artículos recientes, uno de Pope, M., 2000 y otro de Gysbers et al., 1996. Ambos coinciden en las características generales del proceso histórico, pero no en los criterios para organizar el proceso. Mientras Pope se centra en la función y emplazamiento de los servicios de orientación, Gysbers se centra más en el proceso de la institucionalización del concepto.

La orientación para el desarrollo de la carrera es un movimiento eminentemente norteamericano, que hunde sus raíces en lo que en este contexto aconteció y en el surgimiento del movimiento de reforma social. A partir de ese momento, los sucesivos enfoques se han generado en Estados Unidos retomados y adaptados en y a otros lugares. El nombre que ha recibido el trabajo que ha desarrollado un profesional en una institución pública o privada y al que han acudido las personas que han considerado necesario hacerlo para resolver problemas vocacionales, laborales, académicos, familiares o personales o sin localizar exactamente no siempre ha sido el mismo.

En un principio la orientación vocacional fue el término original en EE.UU., a partir del modelo de Parsons. Los términos orientación para la carrera y desarrollo de la carrera aparecen con Super (1955) y con el cambio de denominación de Vocational Guidance Association (1913-1984) a National Career Development Association (1984). A partir de entonces lo que ha sucedido ha sido un debate entre diferentes perspectivas dentro de la orientación para el desarrollo de la carrera (Pope, M.: 2000; Gysbers, N. C.: 1996).

Estos estadios se pueden describir muy brevemente de la siguiente manera (Gysbers, N. C., 1996):

- Periodo de formación, de 1890 a 1950. En este período aparecen en Parsons los conceptos vocación, carrera y ocupación con significados similares, en un momento de cambios sociales ocasionados por el desarrollo industrial y la necesidad de resolución 
de problemas que esto traía consigo. El servicio de contratación creado por Parsons fue en realidad una iniciativa privada. La National Vocational Guidance Association creada en 1913 (hasta 1983 recibió este nombre) define la orientación vocacional como un proceso de ayuda al individuo para la elección y decisión, comprendiendo la planificación y construcción de su futuro laboral. De 1920 a 1939 (Pope, M., Ibid.) gracias a la iniciativa de Weaver, E. y Davis, J. B. la orientación se establece en el sistema educativo primario y secundario (orientación educativa), en el que los procesos de orientación y educación comienzan a ser pensados en uno solo. Brewer en 1932 representa el primer intento de relacionar la orientación y la educación: la orientación y la educación son dos conceptos diferentes en un único proceso de intervención. Para Matewson (1940), la orientación vocacional y la educación se dan en un proceso planificado y estimulante para un desarrollo personal y profesional (Rodríguez Moreno, 1995). A partir de 1940 la orientación llega a los colleges y universidades. Además, hay que contar con el impacto de la II Guerra Mundial y el regreso de los veteranos con el consiguiente problema ocupacional e incorporación de nuevos jóvenes a la universidad (Pope, M.).

- Años de transición, de 1950 a 1980. Los conceptos carrera y desarrollo de la carrera fueron sustituyendo paulatinamente a los de vocación. En 1973, el documento editado por el NVGA define carrera y desarrollo de la carrera como el tiempo de trabajo a lo largo de la vida de una persona. Es interesante hacer notar que es en este periodo cuando surge de manera oficial el concepto de educación para la carrera. A partir de 1974 se institucionaliza el movimiento, siendo Hoydt el director de la Oficina de Educación norteamericana. Por otro lado, a partir de 1960 se da una expansión de la orientación, gracias a la gran cantidad de programas sociales que desde la administración norteamericana se promocionaban para responder así a la demanda entre los jóvenes de trabajo.

- Emergencia de una definición más amplia, de 1980 a 1990. Durante la década de los 70' muchos autores habían situado la ocupación dentro del espectro general de la vida de la persona en cuatro espacios vitales: trabajo, familia, aprendizaje y autoempleo, y papel como ciudadano. En 1976 Super define carrera, incorporando también los diferentes roles de la persona, pero teniendo siempre el trabajo como foco. En 1974, 75 y 81 Gysbers \& Moore definen la carrera y desarrollo de la carrera como "un autodesarrollo a lo largo de la vida en el que interactúan e integran roles, escenarios y eventos de la vida de una persona. En este sentido, la palabra carrera fue identificada con los papeles que la persona desempeña, los escenarios donde se hallan y los eventos planeados o no que ocurren a lo largo de la vida (transiciones). Esta nueva concepción trataba de ampliar la perspectiva laboral que se vino usando hasta entonces para conectarla con una perspectiva más holística y sistémica (Gysbers \& Moore, 1975: Orientación para el desarrollo de la carrera). Gysbers y McDaniels añadieron en 1992 los factores de género, etnia, religión y raza en un esfuerzo por comprender el magma social norteamericano de forma global y actual. En 1985 la NVGA cambió su nombre por el de National Career Development Association, y en 1986 el Vocational Guidance Quarterly pasó a llamarse Career Development Quarterly. A partir de 1980 acontece un cambio de situación, desde la era industrial anterior a la era de la información y coincide con un declinar del sistema económico y la proliferación de servicios de orientación privados. 
La definición anterior se hace realidad. Super en 1990 amplía y perfecciona su teoría de carrera y añade los factores de género, etnia, religión y raza a los espacios vitales, los roles en estos espacios y los eventos de transición y proceso. Características de esta época son el énfasis en la tecnología y los cambios demográficos, la internacionalización de la orientación para la carrera, así como los comienzos de la orientación multicultural y el enfoque de la transición de la escuela al trabajo.

Llegados a este punto lo que interesaría es plantear en qué estado está la teoría sobre el desarrollo de la carrera y la orientación para el desarrollo de la carrera en este momento. La tendencia generalizada es a reorientar la teoría del desarrollo de la carrera en un marco constructivista social, atender al impacto de los cambios económicos, reducir las barreras sociales para el desarrollo de la carrera de grupos específicos como mujeres, grupos minoritarios de origen étnico diverso, orientaciones sexuales diferentes, etc. y replantear la evaluación y el diagnóstico en procesos de desarrollo de la carrera y orientación (Herr, E., 1997).

En el modelo constructivista social (Sexton, Th. L., Griffing, B. L. (Ed.) 1997) se interrelacionan perspectivas como la Biología, los estudios sobre estructura social, la cultura y la identidad en una conjunción dialéctica. En el centro del modelo se encuentra la realidad socialmente construida. En la gramática cultural de la vida diaria, los aspectos físicos, temporales, cognitivos, emocionales, sociales, lingüísticos e intencionales son unificados en una totalidad. No vivimos la cultura, vivimos la realidad. Realidad es algo significativo percibido por personas que interactúan y comparten una gramática cultural.

Las estructuras sociales predisponen a los individuos en la manera de pensar, de actuar y de sentir. Las estructuras sociales imponen una distribución de recursos sociales (autoridad, prestigio, saber, salud, rango, atributos personales, etc.), lo que constituye la dimensión de poder de la vida social. Las relaciones de poder establecidas promueven la reproducción de patrones estructurales de hechos, pensamientos y sentimientos. En el hecho de las relaciones de poder establecidas, los individuos enculturados son menos dados a reconocer sus poderes creativos y más dados a no ver caminos de cambio.

Es lo que Bourdieu (1988) entiende como hábito. El hábito es un mecanismo estructurante que opera en el agente, uniendo a este a su micro-cultura. Está construido como un principio generador, capacitando al actor para actuar en situaciones inseguras, de riesgo y de cambio. Como concepto, el hábito es individualizado y determinativo e implica disposición, tendencia, propensión o inclinación. El hábito como espacio o campo social cambia por medio de dos procesos: la trayectoria social y la conciencia. La educación juega aquí un papel fundamental, internalizando de hecho los hábitos, esto es, los esquemas de percepción, de pensamiento, y acción que proporciona el hábito. Normalmente la gente no tomamos las decisiones laborales ni de manera totalmente racional, considerando todas las opciones, ni ciegamente o bajo la influencia de consideraciones externas. Se toman decisiones según sentido común y prácticas sistematizadas, que es lo que constituye el hábito (inercias). Decidimos mediante hábitos lo que supone el establecimiento de relaciones entre representaciones internas y contextuales. Aplicando este concepto a los "intereses" o las "elecciones", pasamos a enfatizar los intereses como una respuesta a las ocupaciones habituales y dominantes de una sociedad. De esta manera, los intereses pueden ser redefinidos como una manera o forma de estereotipo. Para la orientación constructivista los intereses son emplazados en la localización y diferenciación de experiencias vitales en clientes y espacios sociales a través de hábitos aportados por la conciencia encubierta. 
Las sociedades multiculturales se caracterizan por la persistencia de desigualdades de poder y de acceso a los recursos de que dispone una sociedad altamente estructurada como lo son las sociedades de la información y del conocimiento. La heterogeneidad cultural y estructural de estas sociedades está basada en la herencia (raza y etnia), género, clase socioeconómica, religión, ocupación o edad. Esta diferenciación implica una distribución de contextos culturales, cuyo resultado es la construcción de identidades únicas individuales, cada una enraizada en su origen social, étnico, religioso, de género, etc. Estas diferencias imponen la necesidad de la negociación sobre la construcción de la realidad. Esto es, las identidades actuales son siempre negociadas, no determinadas, debido a la naturaleza abierta de los roles culturales y lo emergente del contexto. Diferentes contextos exigen en una misma persona diversas muestras de su identidad o diversas identidades.

Todo esto induce a una serie de cambios en la comprensión de la carrera: reducción de la distancia entre vida y carrera profesional; los nuevos planteamientos subrayan la importancia de la dialéctica contexto-yo como un fenómeno holístico; actualización de nuestra autopercepción; si la identidad ha aparecido durante décadas estandarizada y cristalizada, en estos momentos se tiende a concebir las identidades como reversibles.

El nuevo paradigma emergente descansa sobre cuatro premisas fundamentales: Los procesos del conocimiento humano son anticipatorios (el Yo como proyecto). Los individuos son auto-organizativos. El ser humano supone una metáfora del ambiente. El Yo se encuentra en continuo desarrollo.

La planificación o proyecto vital (Peavy, 1994) sigue adquiriendo en el paradigma constructivista una consideración fundamental. La orientación constructivista combina la práctica del cliente con sus procesos psicológicos. Desde esta perspectiva se destacan las siguientes características:

- El individuo dispone de la plasticidad suficiente para la reconstrucción del ambiente y de sí mismo. La plasticidad implica un proceso dialéctico, dinámico tanto en el sujeto como en el ambiente, los cuales van transformándose simultáneamente.

- La orientación constructivista subraya la actividad (Young, 1995. ERIC); bajo tres perspectivas:

- como comportamiento,

- como conocimiento consciente,

- y como significado social.

\section{La orientación multicultural}

Otro movimiento surgido en Estados Unidos es la orientación multicultural. D'Andrea y Daniels (1995) la definen como un proceso en el que interactúan un profesional y un cliente con un trasfondo cultural determinado con el propósito de ayudar cognitiva, emocional, psicológica o espiritualmente al cliente. Sus comienzos se hallan ligados a varias organizaciones profesionales, la ACA (American Counselling Association), la APA (American Psychological Association), revistas científicas vinculadas a dichas asociaciones y a la situación multicultural norteamericana, consecuencia de un período de colonización y posteriores movimientos migratorios hacia EE.UU. Como apunta Jackson, M. L. (Ponterotto 1995: 
3 y ss.) el origen de la orientación multicultural responde lógicamente a la necesidad de encarar el problema general de esta situación, en la que el racismo, la segregación, la discriminación y los prejuicios institucionales y organizacionales formaban parte de la cultura dominante.

Como en otros terrenos en la estructura social norteamericana la lucha no sólo por la incorporación y ascensión a puestos de decisión en organizaciones e instituciones, sino también por el disfrute de los servicios públicos y privados educativos y de salud fue lenta para miembros de minorías étnicas hasta la década de los 50 (nacimiento del movimiento), en la que algunos autores publicaron en revistas científicas y la década de los 60 (infancia), década marcada por cambios importantes. En 1964 se publica el Acta de los Derechos Civiles (Civil Rights Act), lo que permite una cierta preocupación por las "culturas en desventaja" y por una mayor permeabilidad en las instituciones en favor de las minorías. Esta década vería nacer en 1965 a la Comisión de Derechos Humanos, así como la ascensión de miembros de minorías étnicas a puestos de gobierno de asociaciones tan importantes como la Asociación Americana de Orientación (APGA hasta 1992).

La década de los 70 es una década marcada por la demanda y circulación de información sobre minorías y grupos tradicionalmente excluidos, como mujeres, personas discapacitadas y otros grupos. En 1970 fue elegido Paul Smith, el primer director negro, de la Personnel Guidance Journal. En 1975 Donald Sue fue llamado por la APGA para ser editor invitado, en 1971 aparece el Guidance Monograph Series 6: Minority Group and Guidance y en 1973 Paul Pedersen dirige una sesión de trabajo sobre orientación cross cultural en la Asociación Americana de Psicología (APA), fruto de la cual aparece el libro Counselling Across Cultures. En 1975 la APA publica la primera guía ética de orientación cross cultural; los profesionales que no fueran competentes a la hora de interactuar con clientes pertenecientes a grupos culturales minoritarios serían tachados de no éticos. Esta guía sería el embrión del posterior desarrollo de competencias profesionales en el campo de la orientación multicultural.

El crecimiento en investigación y publicaciones sobre multiculturalidad en orientación durante las décadas de los ' 80 y ' 90 es enorme. La oferta de curricula conteniendo multiculturalidad ha crecido del 1\% en 1977 al 89\% en 1992; sin embargo, el campo de la orientación es monolítica, en parte porque su desarrollo se hace desde una perspectiva anglo-europea. Esto se demuestra en las posiciones de poder en las organizaciones antes mencionadas y en el número de orientadores blancos en su mayoría (D’Andrea \& Daniels: 23). A la vista de lo manifestado por estos autores, los cambios en materia de orientación multicultural deben venir desde la misma gestión de las estructuras de poder (consejos de gobierno, juntas consultivas, comités ejecutivos) de forma que estimule cambios sistemáticos en las instituciones profesionales (estrategias de movilización, educativas, organizacionales e institucionales).

\section{Modelo de competencias interculturales en servicios de orientación para el desarrollo de la carrera en universidades a distancia en Europa}

La Unión Europea es ya una realidad; una realidad llena de incertidumbres, esperanzas y dudas. Sobre cuatro libertades se asienta la Unión: libertad de comercio, libertad de movi- 
miento de todos los ciudadanos europeos, libertad de circulación de capital y de servicios (Omnia, 1996). Hay tres modos de comprender la diversidad que se da en Europa: por un lado, la diversidad dentro de cada Estado Nación: género, etnia, religión, clase social, orientación sexual son los principales factores a tener en cuenta. Por otro lado, los ciudadanos de estos países pueden moverse de un país a otro sin problemas dentro de las fronteras europeas, buscando nuevos espacios laborales o de formación. Por último, pueden comprenderse a aquellos ciudadanos que vienen de fuera de los confines europeos. Una característica de esta nueva situación es su tendencia a la homogeneidad y convergencia cultural, por un lado, y por otro la tendencia a la diversidad. Lo que parece que están surgiendo son nuevas estructuras de diferencias comunes (Wilk, 1995, en Paul du Gay 1997).

Dentro de este contexto multicultural las Universidades a Distancia tienen la posibilidad de poseer la virtud de la flexibilidad que desde la esfera económica y política se consigna como necesaria: pueden traspasar las fronteras nacionales, pero sin dejar de tener un carácter nacional. La Orientación dentro de la estructura universitaria se perfila como un servicio de ayuda al desarrollo de la carrera de los individuos.

Independencia y autonomía del usuario (Wedemeyer, Charles A., en Keegan, D., 1983), industrialización de la enseñanza (Peters, O., 1974 en Keegan, Desmond, 1983), e interacción y comunicación (Baath, Holmberg, Daniel, Sewert, Smith, en Keegan, Desmond, 198) son los tres pilares teóricos que se manejan a la hora de articular servicios, procesos y productos dentro de la educación a distancia actuales. Ahora bien, si comprendemos la educación a distancia como un sistema cultural (Hall, Stuart, 1997) la entenderemos como la producción, circulación (comunicación), consumo y regulación (certificaciones) de significados a través de programas, actividades y experiencias educativas principalmente no presenciales o a distancia, tanto formales para programas educativos graduados, como no formales para aquellos que no son graduados. Estos programas utilizan representaciones diversas para su circulación mediante textos, imágenes y sonidos, e inciden de manera significativa en la identidad de sus consumidores.

A pesar del gran desarrollo tecnológico, los centros universitarios siguen requiriendo de una descentralización presencial a cargo de centros de estudio que hagan posible una cierta contextualización y circulación de los programas, el desarrollo de trabajos de grupos y dé respuestas adecuadas de forma no estandarizada a dudas y cuestiones que difícilmente de otro modo serían posible. Los servicios de orientación al estudiante se insertan prioritariamente en estos centros, aunque también utilicen medios de comunicación para ello (Clennell, S, Peters, J \& Sewart, D., en: Sewart et al.: 1983) y se hallen en las centrales de las instituciones. Este es un denominador común a todas las instituciones universitarias hasta ahora visitadas y consultadas.

Si se consultan los datos que proporcionan las estadísticas de cada localidad, estos contextos albergan una gran diversificación tanto en el alumnado potencial como el real. Aún sin consultar ningún dato estadístico alguno, nada mejor que pasear por las calles de algunas de las ciudades que cuentan con centro de estudios para observar gentes de todas las procedencias, de todas las capas sociales, de todas las edades, etc. Las instituciones universitarias a distancia consultadas son instituciones que predican una función pública sin discriminación alguna. Los usuarios de estos centros tienen derecho a una atención competente, que tenga en cuenta sus características y necesidades peculiares. 
Como queda claro en la revisión de fuentes más actuales, no se pueden entender los procesos educativos, sociales, políticos o económicos fuera de un marco global multicultural. En nuestro discurso, hablar de competencias interculturales de los profesionales que trabajan en instituciones universitarias abiertas o a distancia requiere hablar de competencias interculturales de la misma institución. La cultura de la institución es fundamental para el comportamiento e identidad de las personas que trabajan en ellas, dejando aparte la influencia de toda la estructura sociopolítica en la que las instituciones se hallan. Por ello es necesario comprender que las fuerzas organizacionales e institucionales pueden impedir o potenciar el desarrollo de las competencias interculturales. Sin ánimo de agotar todas las posibilidades, estas son las características de una organización inclusiva y culturalmente competente:

1. La política, misión y visión multiculturales están explicitadas por escrito.

2. Se da un plan de acción multicultural y para la diversidad.

3. El equipo de trabajo es multicultural.

a) Se fomentan metas comunes.

b) La diferenciación de roles están claramente especificada.

c) Se formalizan relaciones específicas en torno a tareas y experiencias comunes.

d) Los procesos de gestión se desarrollan a partir de

i. la definición de la actividad colectiva

ii. sentido colectivo

iii. autorreflexión como grupo.

e. Desarrollo de una cultura común en torno a conjunto de valores y normas comunes, previamente consensuadas.

4. La organización solicita feedback de los grupos de empleados.

5. Incluye criterios de competencias multiculturales en la evaluación del sistema.

6. Provee de mentorización y apoyo a las redes de empleados pertenecientes a los diferentes grupos culturales.

7. Fomenta la formación de coaliciones y redes de apoyo.

8. Hay un compromiso a largo plazo en la formación permanente.

9. La organización es reflejo de una comunidad amplia.

En cuanto a las competencias interculturales de los profesionales de la orientación, éstas comprenden tres áreas: una reflexión sobre nosotros mismos que nos haga conscientes de nuestros propios valores, prejuicios y asunciones. La consideración de los otros, que nos acerque su universo cultural y a las situaciones en las que viven. Por último, un desarrollo estrategias y procedimientos culturalmente adecuados. Cada competencia comprende una dimensión actitudinal, otra cognitiva y otra comportamental. Se indican a continuación las competencias más importantes a ser desarrolladas por los orientadores:

- Dimensión A) Conciencia de las propias asunciones, valores y prejuicios

- Reflexión y conciencia del propio universo y transfondo cultural, así como de la propia identidad cultural (actitudinal). 
- Reflexión y conciencia de la influencia de lo anterior en la relación con los usuarios (actitudinal).

- Conocimiento del sistema de valores inherente a las teoría manejadas por el profesional, y qué valores son inherentes a las estrategias y técnicas usadas en la situaciones interactivas al nivel profesional específico? (cognitivo).

- Conocimiento del impacto de los estilos de comunicación en el cliente (cognitivo).

- Implicación en experiencias multiculturales (comportamental).

- Dimensión B) Comprensión del universo cultural de los clientes

- Reflexión en torno a los estereptipos, percepciones y creencias sostienen acerca de los grupos culturalmente diferentes que supongan un impedimento de su habilidad para llevar a cabo una relación profesional? (actitudinal).

- Conocimiento del universo cultural de los usuarios y de su impacto en las carreras de los usuarios (cognitivo).

- Conocimiento de las influencias sociopolíticas en los diferentes grupos, en especial los procesos y situaciones de discriminación que viven ciertos grupos culturales (cognitivo).

- Ser capaces de estar al corriente de la investigación multicultural relevante (comportamental).

- Dimensión C) Desarrollo de estrategias de intervención apropiadas

- Respeto y colaboración con grupos, asociaciones culturalmente diferente (actitudinal).

- Conocimiento del posible poder discriminatorio tanto de los procesos de atención al usuario, como de las prácticas institucionales (cognitivo).

- Ser capaz de interactuar diferenciadamente según el patrón cultural del cliente (comportamental).

\section{Competencias en servicios de orientación para el desarrollo de la carrera en universidades a distancia en Europa a la luz de la investigación}

En una investigación cualitativa recientemente finalizada (Castellano, J. L. 2005) usando como método de recogida y análisis de datos la entrevista, los grupos de discusión abierta y el análisis cualitativo de contenido (Ballastaed et al., 1981) se investigó la percepción de los factores incidentes en el desarrollo de la carrera de los orientandos.

Cinco universidades europeas fueron visitadas: UNED, FernUniversität alemana, Open University británica, Open Universiteit holandesa y Universidade Aberta portuguesa. La investigación se enfocó los niveles institucional y profesional. Concretamente se consultaron 4 responsables de servicios centrales de orientación y 1 a nivel EADTU (European Association Distance Teaching Universities), asociación donde se encuadran las universidades objeto de análisis, 13 coordinadores de centros asociados europeos y se organizaron 7 grupos de discusión con orientandos. 
En septiembre de 2002, y tras someter a proceso de validación los instrumentos de recogida de datos, se procedió a su implementación en una prueba piloto en la sede central del COIE de UNED en Madrid. De octubre a noviembre de 2002 se visitó la FernUnviersität, de enero a abril de 2003 la el resto de los centros de la UNED y la Universidade Aberta y de abril a julio del mismo año la Open Universiteit y la Open University.

De los documentos institucionales publicados se desprende que únicamente la Open University explicita como una de sus líneas estratégicas la inclusión de grupos culturales (Plans for Change. The University's strategic and development plans 2002-2012), incluyendo dentro de estas medidas el reclutamiento y retención de miembros de grupos culturales: minorías étnicas, discapacitados, grupos de procedencia de estratos socioculturales bajos, y grupos con resultados académicos bajos. También el apartado de investigación incluye la financiación de investigación para los servicios de apoyo al estudiante, esto es, los servicios de orientación. En las demás universidades no hay una política que comprenda la diversidad cultural ampliamente entendida (género, origen étnico o nacional, situación de migración, religión, edad, religión, estilos cognitivos, de aprendizaje, grupos con necesidades especiales, etc.).

Las instituciones universitarias a distancia investigadas son heterogéneas en lo que se refiere a su organización, lo que trae consigo una gran diversidad en cuanto a las líneas de orientación llevadas a cabo, la coordinación de procesos de evaluación de necesidades, el fomento de procesos de formación permanente. Ninguno de estos apartados citados son llevados a cabo, a excepción de las líneas de orientación que evidentemente todas las universidades siguen en una u otra dirección. Ninguna institución consultada tiene en cuenta variables culturales de manera sistemática, esto es, planteándose un política multicultural, a la hora de seleccionar el equipo profesional. Esto tiene cierta lógica, ya que no se parte de una análisis en profundidad de necesidades contextuales, lo que deviene en parte de la teoría clásica que subyace a las instituciones a distancia, esto es, predominio de la estandarización y mecanización productiva a todos los niveles, en este caso también a nivel del concepto de orientación ofrecido.

En el contexto de la FernUniversitaet se perciben como factores determinantes del desarrollo de la carrera sobre todo el estatus socioeconómico, el sistema educativo y la tradición en la que se funda la universidad. En segundo plano quedan factores culturales más personales, como la religión y el trasfondo cultural. Estos factores reproducen y exageran la condición social de partida de las personas, además suponen de por sí una limitación bastante clara de inclusión.

A la hora de prestar ayuda orientadora, el profesional orientador que trabaja en la FernUniversitaet percibe que queda afectado sobre todo por el sistema educativo, la experiencia académica previa y el trabajo. En segundo lugar, el orientador queda influido por su estatus socioeconómico, su ambiente familiar, la edad y la vida privada. Estos factores suponen la base organizativa de la oferta cultural, también supone una plataforma para la profundización de las fases del proceso de orientación: sistema de aprendizaje y metodología y, por último, como elementos conformantes de los procesos de orientación dirigidos especialmente hacia la igualdad en los procesos de orientación según el carácter de cada uno, así como para armonizar estudios, trabajo y vida privada.

Contexto OUNL. En el contexto holandés de la OUNL los factores considerados importantes para el desarrollo de la personalidad son el estatus socioeconómico (entendidos como 
limitadores) y el político, en el caso de los refugiados. A pesar de ello, hay siempre alguien que opina que no tienen importancia en la OUNL. El orientador percibe que queda también afectado en su comportamiento comunicativo.

Contexto UKOU. En el contexto británico, aunque se comprenden los motivos personales como posibilitadores de superación de barreras culturales, se perciben la nacionalidad, el género, el estatus socioeconómico o la religión como muy importantes para el desarrollo de la personalidad y el aprendizaje intelectual. Cuando se tiene una mentalidad abierta se aprende mejor y se recibe mejor la orientación.

De igual modo, una mentalidad abierta en el orientador permite la comprensión de las diferencias culturales en situaciones particulares, las expectativas personales representan el punto de partida de las sesiones de orientación.

En el contexto UNED se percibe que la lengua de trabajo genera problemas de comunicación si no se domina, la escala de valores y las barreras por pertenencia de género (femenino), determinan objetivos y el grado de implicación en la tarea, limitando el acceso al mundo laboral y generando desigualdades retributivas; la religión, el origen nacional, el estatus socioeconómico generan diferencias en el desarrollo de la personalidad, diferencias en la gestión del tiempo de carrera y el grado de dedicación al estudio, diferenciando roles, diferenciando las perspectivas de futuro y limitando el proyecto de carrera. Las diferencias tipológicas de personalidad, generan diferencias en estilos de aprendizaje y elecciones académicas.

En cuanto a la influencia de los factores culturales a la hora de prestar orientación se citan la actividad del servicio de orientación se percibe neutra culturalmente, suponen factores causales para incentivar el proceso de ayuda, cuando suponen limitaciones de comprensión, causan una orientación individualizada, el problema de las barreras por pertenencia de género se refleja en el programa de orientación, no se tolera el papel de la mujer en la religión musulmana.

Contexto Aberta. Los factores culturales, excepto la religión, afectan al desarrollo de la carrera de las personas: La pertenencia de género estadísticamente en la presencia en la universidad, el origen regional afecta al comportamiento social y éste es valorado diferencialmente por empresas e instituciones, la situaciones de extranjería y de refugio político son valoradas negativamente por los medios de comunicación. En el caso de la orientadora, por un lado, no se da una influencia personal de factores culturales, pero en otros casos, es importante la forma de la comunicación para comprender el origen de los orientandos. Se estima importante evitar el ruido comunicativo debido a la presencia inconsciente de la historia personal y la experiencia.

Generalmente los orientadores responden a este Item guiados por la información recogida a partir de medios de comunicación y de la propia intuición, ya que no se dispone de base de datos construida a tal efecto.

\section{Conclusiones}

El modelo de competencias interculturales en servicios de orientación para el desarrollo de la carrera de los ciudadanos que conviven en alguno de los países europeos mencionados y su institucionalizacón e internalización en los profesionales trata de erradicar los prejui- 
cios y discriminación por razón de género, procedencia étnica, situación social, religión y cualquier otro factor que haga a un grupo o a personas diferentes. La Universidad a Distancia o Abierta puede salvar barreras largamente soportadas por determinados grupos y personas, y con ello asegurar, por un lado la igualdad de oportunidades de elección de carrera vital digna, y por otro asegurar la flexibilidad que la situación exige a todas las instituciones responsables de la vida en convivencia.

A la luz de la investigación llevada a cabo, a nivel institucional no se lleva a cabo ninguna medida que contextualice culturalmente las universidades en sus entornos más inmediatos y específicos, allí donde van a ser reconstruidos los programas elaborados desde las centrales. Desde esta perspectiva poco podrán los profesionales de la orientación para ayudar a la construcción de las carreras de los orientandos que se la solicitan, ya que se carece de los instrumentos necesarios para, en primer lugar, saber las necesidades de los usuarios de los servicios y, en segundo lugar, tampoco hay una actitud abierta a las posibilidades que ofrecen los entornos en orden a proporcionar a aquellos la formación e información necesaria para un desarrollo fructífero de sus carreras.

Para ello sería necesario, a nivel de investigación, una colaboración interinstitucional, analizar exhaustivamente tópicos como la identidad cultural, procesos emocionales, estereotipias y prejuicios y comunicación intercultural y grado de percepción de influencias de contextos sociopolíticos en el desarrollo de las carreras de personas pertenecientes a grupos culturales minoritarios o discriminados históricamente. A nivel institucional central comprender una política multicultural, la elaboración de programas de formación en competencias multiculturales, un equipo profesional multicultural, incentivar la investigación coordinada con centros asociados (análisis de necesidades) y elaborar representaciones institucionales incluyentes. A nivel de centros la implicación en programas de investigación para saber las necesidades de la zona, la exploración previa y colaboración con asociaciones y grupos culturales, elaboración de programas de formación en competencias interculturales, evaluación o retroinformación continua y una política de colaboración intereuropeo. A nivel profesional se hace necesario una formación en comunicación intercultural, desarrollo de la identidad multicultural y sobre las técnicas y destrezas culturalmente adecuadas, sobre todo despertar en la conciencia que los instrumentos hasta ahora construidos están anclados en tradiciones culturales específicas.

\section{Referencias bibliográficas}

Aguado, T., Gil, J. A., Jiménez, R. y Sacristán, A. (1998). Diversidad cultural e igualdad escolar. Un modelo para el diagnóstico y desarrollo de actuaciones educativas en contextos escolares multiculturales. MEC. Madrid.

Arnold, (1989). En Glagow et al.: Theoretische Überlegungen zur entwicklungspolitischen Professionalität und Handlungskompetenz. Tagungsbericht. Zir. N. Arnold 1989: 6.

Arredondo, P., Toporeck, R., Back, Sh., Locke, Don C., Jones, J., Sánchez, J. \& Stadler, H. (1996). "Operationalisation of the Multicultural Counselling Competencies". Journal of Multicultural Counselling and Development. V. 24 N. 1 ACA

Auernheimer, G. (1999). Einführung in die Interkulturelle Pädagogik. Vorlesung - Dokumente. Universität zu Köln. 
Auernheimer, G. (2002). Interkulturelle Kompetenz und pädagogische Professionalität. Leske + Budrich. Opladen.

Baitsch, Ch. (1996). Kompetenz von Individuen, Gruppen und Organisationen. TU Chemnitz-Zwickau.

Baitsch, Ch. (1985). Kompetenzentwicklung und partizipative Arbeitsgestaltung. Peter Lang

Ballaestaed, S.-P., Mandl, H., Schnotz, W. \& Tergan, S.-O. (1981). Texte verstehen, Texte gestalten. Urban \& Schwarzenberg. München.

Berger, P. L. \& Luckmann, Th. (1984). La construcción social de la realidad. Buenos Aires. Amorrortu

Bourdieu, P. (1988). La distinción: criterios y bases sociales del gusto. Madrid, Taurus.

Callejo, J., Aguado, T., Ballesteros, B.,Gil, I. y López, B. (2001). "Indicadores de evaluación de la educación a distancia en un sistema universitario". RIED, Vol. 4, N. 1.

Castellano Pérez, J. L. (2005). Competencias en servicios de orientación de universidades a distancia o abiertas en Europa desde una perspectiva intercultural. UNED. Tesis doctoral.

Dekelskamp (1991). "Die Simulation als Medium zum interkulturellem Lernen". En Nestvogel, R. (Ed.): Interkulturelles Lernen oder verdeckten Dominanz.

D'andrea, M. \& Daniels, J. (1995). "Promoting Multiculturalism and Organisational Change in the Counselling Profession. A Case Study". En Pontoretto et al. (1995): Handbook of multicultural counselling. Thousand Oaks, California. SAGE.

Du Gay, P. (1997). Production of Culture / Cultures of Production. Sage \& Open University.

Essinger (1986). "Interkulturlle Pädagogik”. En Borelli, 1986: Interkulturelle Pädagogik. Baltmannsweiler.

Fuertes, J. N. (2001), Bartolomeo, M. \& Nichols, M. (2001). "Future Research Directions in the Study of Conselor Multicultural Competency". En Journal of Multicultural Counseling and Development. ACA.

Gysbers, N. G. (1996). "Beyond Career Development - Life Career Development Revisited”. En Feller, R. \& Walz, G.: Career Development in Turbulent Times. ERIC 11-30.

Hall, S. \& Gieben, Br. (1992). The Formation of Modernity. Polity Press in Association with the Open University.

Hall, Stuart (1997). Representation. Cultural Representations and Signifying Practices. SAGE-Open University. London.

Hammer, G., Wiseman (1978). "Dimensions of intercultural effectiveness: An exploration study". En International Journal of Intercultural Relations.

Hannigan (1991). "Traits, attitudes, and skills that are related to intercultural effectiveness and their implications for cross-cultural training”. En International Journal of Intercultural Relations.

Herbrand, Fr. (2000). Interkulturelle Kompetenz. Grundlagen - Interkulturelles Training in Theorie und Praxis - Handlungsempfelungen. Paul Haupt Verlag. Bern, Stuttgart, Wien.

HERR, Edwin L. (1997). Super's Life-Span, Life-Space Approach and Its Outlook for Refinemten. En: Savickas, Mark L. (editor) (1997). Special Section; Advancing Life-Span, Life-Space Theory. The Career Development Quartely. Vol. 45, N. 3

Hoffmann, E. (2002). "Das TOPOI Modell - eine Heuristik zur Analyse interkultureller Gesprächssituationen und ihre Implikationen für die Pädagogische Arbeit”. En Auernheimer (2002). Interkulturelle Kompetenz und pädagogische Professionalität. Leske + Budrich. Opladen.

Keegan, D. (1983). Six Distance Education Theories. Fern Folleto 042. ZIFF. Fernuniversität Hagen. 
Kolb, D. A. (1984). Experimental Learning. Experience as the Source of Learning and Development. Prentice-Hall, New Jersey.

Leontjew, A. N. (1973). Probleme der Entwicklung des Psychischen. Fischer. Frankfurt.

Lave, J. \& Wenger, E. (1993). Situated Learning. Legitimate Peripheral Participation. Cambridge University Press. N.Y.

Martin Bris, M. (2001). La calidad educativa en un mundo globalizado: intercambio de experiencias y perspectivas.

OCDE, (1991). Escuelas y calidad de la enseñanza. Informe International. Paidós - MEC, Madrid.

Palmer, C. \& Laungani, P. (1999). Counselling in a multicultural society: SAGE.

Peters, O. (1973). Die didaktische Struktur des Fernunterrichts. Untersuchungen zu einer industrialisierten Form des Lehrens und Lernens. Weinheim: Beltz.

Ponterotto, Joseph et al. (1995). Handbook of multicultural counselling. Thousand Oaks, California. SAGE.

Pope, M. (2000): “A Brief History of Career Counselling in the United States". The Career Development Quartely.

IUED (2000). Proyecto Brial. UNED.

Ruben, (1990). "The study of cross-cultural competence: Traditions and contemporary issues". In International Journal of Intercultural Relations.

Savickas, M. L. (Ed.) (1997). "Special Section; Advancing Life-Span, Life-Space Theory”. The Career Development Quartely. Vol. 45, N. 3.

Sexton, Th. L. \& Griffing, B. L. (Ed.) (1997). Constructivist Thinking in Counseling Practice, Research, and Training. Teachers College Press, 1234 Amsterdam Ave., NY.

Sewart, D. et al. (1983). Distance Education: International perspectives. Croom Helm.

Sodowsky, G. R., Taffe, R. C., Gutkin, T. B. \& Wise, S. L. (1994). "Development of the Multicultural Counseling Inventory: A self report measure of multicultural competencies". Journal of Counselling Psychology, 41: 137-148

Sodowsky et al. (1996). 3 y ss. En Pope-Davis, y Coleman: Multicultural Counselling Competencies. Assessement, Education and Training and Supervision. SAGE.

Sue, W., Arredondo, P., Mcdavis, R. (1995). "Multicultural Counseling Competencies and Standards: A Call to the Proffesion". En Ponterotto 1995: Handbook of Multicultural Counseling. SAGE: 624-645.

Sue, D. W., Carter, R. T., Casas, J. M., Fouad, N. A., Ivey, A. E., Jensen, M., Lafromboise, T. \& Manese, J. E. (1998). Multicultural Counseling Competencies. Individual and Organizational Development. SAGE.

Sue \& Sue (1999). Counseling the culturally different. Theory and Practice. John Wiley \& Sons, Inc. N.Y., Toronto: 214 y ss.

Watts, A.G. \& Van Esbroeck, R. (1998). New Skills for New Futures: Higher Education Guidance and Couselling. VUPRESS and FEDORA.

Wenger, E. (1998). Communities of practice. Learning, Meaning and Identity. Cambridge University Press. N.Y. 